\title{
Cost-effectiveness analysis of FOLFIRINOX vs gemcitabine with nab-paclitaxel as adjuvant treatment for resected pancreatic cancer in the United States based on PRODIGE-24 and APACT trials
}

Aditi A Kharat, BPharm, MS; Richard Nelson, PhD; Trang Au, MPH, PharmD; and Joseph Biskupiak, PhD, MBA

\section{What is already known about this subject}

- Prognosis for pancreatic cancer patients is poor, and the overall survival is low.

- FOLFIRINOX and gemcitabine with nab-paclitaxel (GemNab) offer increased median survival duration for pancreatic cancer compared with gemcitabine monotherapy.

- FOLFIRINOX and GemNab are the recommended standard of care for pancreatic cancer patients with a good ECOG performance status.

\section{ABSTRACT}

BACKGROUND: Pancreatic cancer is associated with low median overall survival. Combination chemotherapy regimens FOLFIRINOX and gemcitabine with nabpaclitaxel (GemNab) are the new adjuvant treatment standards for resectable pancreatic cancer. PRODIGE-24 and APACT trials demonstrated superior clinical outcomes with FOLFIRINOX and GemNab, each vs gemcitabine monotherapy.

OBJECTIVE: To evaluate the cost-effectiveness of FOLFIRINOX vs GemNab for resectable pancreatic cancer in adults from

\author{
What this study adds \\ - This study was the first to perform a \\ cost-effectiveness analysis comparing \\ FOLFIRINOX and GemNab as adjuvant \\ therapy for resected pancreatic cancer. \\ - FOLFIRINOX was not cost-effective \\ at a willingness-to-pay threshold of \\ $\$ 200,000$ per quality-adjusted life-year. \\ - FOLFIRINOX was more effective \\ than GemNab; however, the increase \\ in effectiveness was associated \\ with increased costs in the relapse- \\ free survival state, which may be \\ attributable to the increased toxicity of \\ FOLFIRINOX, and the costs associated \\ with management of those adverse \\ events.
}

the U.S. payer perspective, in order to inform decision makers about which of these treatments is optimal.

METHODS: A Markov model with 3 disease states (relapse free, progressive disease, and death) was developed. Cycle length was 1 month, and time horizon was 10 years. Transition probabilities were derived from PRODIGE-24 and APACT survival data. All cost and utility input parameters were obtained from published literature. Costeffectiveness analysis was performed to obtain total costs, quality-adjusted life-years (QALYS), life-years (LYs), and incremental cost-effectiveness ratio (ICER). A 3\% annual discount rate was applied to costs and

\author{
Author affiliations \\ Aditi A Kharat, BPharm, MS; Richard Nelson, \\ PhD; Trang Au, MPH, PharmD; and Joseph \\ Biskupiak, PhD, MBA, Department of \\ Pharmacotherapy, University of Utah, \\ Salt Lake City. \\ AUTHOR CORRESPONDENCE: \\ Aditi A Kharat, 415.619.8669; \\ aditi.kharat@utah.edu \\ J Manag Care Spec Pharm \\ 2021;27(10):1367-75 \\ Copyright $\odot 2021$, Academy of Managed \\ Care Pharmacy. All rights reserved.
}

outcomes. The effect of uncertainty on model parameters was assessed with 1-way and probabilistic sensitivity analysis (PSA).

RESULTS: Our analysis estimated that the cost for FOLFIRINOX was $\$ 40,831$ higher than GemNab (\$99,669 vs. $\$ 58,837)$. Despite increased toxicity, FOLFIRINOX was associated with additional 0.18 QALYs and $0.25 \mathrm{LYs}$ compared with GemNab (QALY: 1.65 vs. 1.47; LY: 2.09 vs. 1.84). The ICER for FOLFIRINOX vs GemNab was $\$ 226,841$ per QALY and $\$ 163,325$ per LY. FOLFIRINOX was not costeffective at a willingness-to-pay (WTP) threshold of $\$ 200,000$ per QALY, and this was confirmed by the PSA. 
CONCLUSIONS: Total monthly cost for FOLFIRINOX was approximately 1.7 times higher than GemNab. If the WTP threshold increases to or above $\$ 250,000$ per QALY, FOLFIRINOX then becomes a cost-effective treatment option.
Pancreatic cancer is the third most common cancer and the third leading cause of cancer-related death in the United States. ${ }^{1,2}$ It is estimated that approximately 60,430 people will be diagnosed with pancreatic cancer, and 48,220 deaths will be attributed to it in $2021 .^{3}$ Exocrine tumors in the pancreas cause adenocarcinoma, the most common type of pancreatic cancer. ${ }^{4}$ Surgical resection is the preferred firstline treatment for pancreatic cancer, given the improved survival outcomes. ${ }^{5}$ Patients diagnosed with advanced disease are typically not candidates for surgery. ${ }^{5,6}$ For resected pancreatic cancer, evidence suggests treatment with adjuvant chemotherapy achieves longer survival. ${ }^{6-8}$

FOLFIRINOX (combination of 5 fluorouracil, leucov $\neg$ orin, oxaliplatin, and irinotecan) or gemcitabine with nabpaclitaxel (GemNab) are the recommended adjuvant chemotherapy regimens for pancreatic cancer., ${ }^{9,10}$ In the phase 3 PRODIGE-24 study, patients diagnosed with pancreatic cancer who underwent R0 or R1 resection were randomized to either FOLFIRINOX or gemcitabine monotherapy and evaluated for disease-free survival (DFS), overall survival (OS), and toxicity. Results indicated significantly longer OS and DFS in patients treated with FOLFIRINOX vs gemcitabine (DFS: 21.6 months vs 12.8 months, hazard ratio $[\mathrm{HR}]=0.58[0.46-0.73]$; OS: 54.4 months vs 35 months, $\mathrm{HR}=0.64$ [0.48-0.86]). The incidence of grade $3 / 4$ adverse events (AE) was higher in the FOLFIRINOX group (Supplementary Table 1, available in online article). ${ }^{7}$

APACT, another phase 3 study, evaluated R0- or R1-resected patients randomized to either adjuvant GemNab or gemcitabine for DFS, OS, and safety outcomes. Preliminary results demonstrated that patients treated with GemNab had improved OS (40.5 months vs 36.2 months, $\mathrm{HR}=0.82$ [0.68-1.00]) and marginal DFS benefit (median 19.4 months vs 18.8 months; HR=0.88 [0.73-1.06]). ${ }^{8}$ PRODIGE-24 and APACT have shown that FOLFIRINOX or GemNab is superior compared with gemcitabine monotherapy for OS with resected PC. However, the cost-effectiveness of these adjuvant chemotherapy regimens is still unknown.

Given the high costs associated with pancreatic cancer and the cancer-related economic burden to the US health care system, ${ }^{11,12}$ understanding the economic impact of therapies relative to their clinical efficacy is important for patients, clinicians, and policymakers. Our research objective was to evaluate the cost-effectiveness of adjuvant treatments (FOLFIRINOX vs GemNab) for adults with resectable pancreatic cancer from the US payer perspective.

\section{Methods}

\section{PATIENT POPULATION}

The patient population from the 2 trials were similar, and a table reporting the clinical and demographic characteristics of the population for the trials is included in Supplementary Table 3 (available in online article). The average age for participants in both trials was 63 years, with the majority of the population being males (PRODIGE: 57.5\%; APACT: $52.8 \%$ ). With respect to Eastern Cooperative Oncology Group (ECOG) performance status, PRODIGE and APACT showed some variations, which suggest that slightly healthier patients received GemNab as opposed to FOLFIRINOX (ECOG performance status 0: 49.8\% vs 58.3\%).

\section{MODEL STRUCTURE}

A Markov model was developed to perform a cost-effectiveness analysis (CEA) between adjuvant chemotherapy regimens using data from the PRODIGE-24 and APACT trials. This model was developed based on a literature review and consultation with an oncology-trained clinical pharmacist with health outcomes research experience. Three mutually exclusive health states were included in the model: relapse-free survival (RFS), progressive disease (PD), and death. After starting in the RFS state, during each cycle patients could transition to either the PD stage or death or remain in the RFS stage. If patients transitioned to the PD state, then they could either remain in the PD state or die (Figure 1). The model cycle length was 1 month, and the time horizon was 10 years.

\section{MODEL INPUTS}

Transition Probabilities. Parametric modeling of survival analysis data was done to determine transition probabilities. First, individual patient-level data were recreated for OS and DFS from Kaplan-Meier curves of PRODIGE-24 and APACT trials. The Kaplan-Meier curve for the PRODIGE-24 trial was obtained from its published study. Since the APACT trial data are not yet published, Kaplan-Meier curves were obtained from data described in a conference presentation. ${ }^{13}$

Second, WebPlotDigitizer software (version 4.2; https:// apps.automeris.io/wpd/index.zh_CN.html) was used to obtain DFS and OS probabilities from the Kaplan-Meier curve of phase 3 trials. Then, the survival data obtained were used to fit the parametric survival model using an 

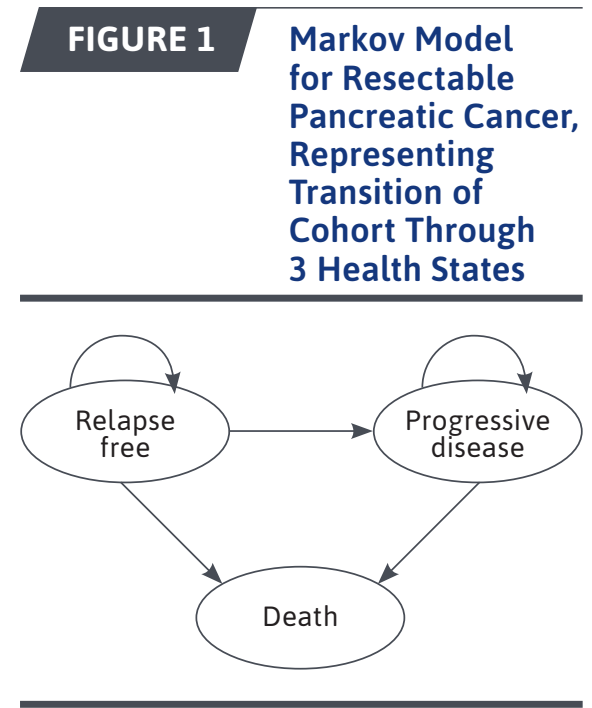

algorithm derived by Hoyle et al. ${ }^{14}$ This enabled us to generalize the findings from the trials and extrapolate survival beyond the data horizon.

Finally, the results of a Weibull fitted model were used to determine the time-dependent transition probabilities. The probability of time-dependent mortality (death) was calculated using the following formula:

$$
\begin{gathered}
\mathrm{p}(\mathrm{T} \rightarrow \mathrm{T}+1)=1-\mathrm{EXP}(\text { lambda } \times \\
(\mathrm{T} \wedge \text { gamma }-(\mathrm{T}+1) \wedge \text { gamma }))
\end{gathered}
$$

$\mathrm{T}$ is the current cycle in the Markov model. Lambda ( $\lambda$ ) and gamma $(\gamma)$ are the Weibull parameters derived from the Weibull curve that closely resembles the survival function (Supplementary Table 1 and Supplementary Figure 2, available in online article). ${ }^{15}$

Costs. Costs associated with acquiring and administering chemotherapy treatments, basic supportive care, inpatient, outpatient, pharmacy, and managing AEs were used to calculate the total costs associated with FOLFIRINOX and GemNab. ${ }^{16-19}$ These costs were obtained from Kim et $\mathrm{al}^{16}{ }^{16}$ who performed a retrospective analysis of hospital claims data supplemented with detailed billing data for inpatient and hospitalbased outpatient services from the MedAssets database, which contains medical administrative data from approximately 350 hospitals in academic and community settings across the United States. ${ }^{16}$

Chemotherapy-related costs included the costs of acquisition and administration of chemotherapy drugs. Outpatient costs included clinical evaluation, emergency department visits, imaging, laboratory tests, radiation, surgery, and other outpatient costs that did not include chemotherapy-related or $\mathrm{AE}$ management cost. ${ }^{16}$

Supportive care costs were defined as the costs of medications to prevent or treat AEs, including neutropenia, anemia, thrombocytopenia, and nausea/vomiting. Supportive care costs included granulocyte colonystimulating factor, blood products, antiemetics, fluids in addition to electrolytes and nutrition, anticoagulants, atropine, pain management medications, and other supportive care expenses. ${ }^{16}$

Since we assumed that major AEs would occur in the first cycle, we used $\mathrm{AE}$ management costs in the first cycle. In the remaining cycles we included supportive care costs and not the $\mathrm{AE}$ management costs. Therefore, we believe double counting was not an issue. Costs associated with progressive disease and death were also included. ${ }^{20,21}$

All cost input parameters were converted to 2020 US dollars using the Consumer Price Index (Table 1).22,23 Health-related quality of life (HRQoL) for each health state is represented by a utility value that ranges from 0 (death) to 1 (perfect health).

Utilities. Utility and disutility values for this study were obtained from previous published studies. The base-case value for RFS state and PD state were 0.85 and 0.73 , respectively. ${ }^{24,25}$

The disutility values for grade $3 / 4$ AEs are shown in Table 2 and are based on expert opinion and literature review. We identified top AEs that have a significant impact on clinical outcomes. Treatment-related grade $3 / 4$ diarrhea, fatigue, and neutropenia occurred in more than $10 \%$ of the patients, and although neutropenia and vomiting occurred in lower proportions of patients ( 5\%), it still was considered to be clinically significant based on expert opinion.?

We derived a weighted disutility value for each intervention by multiplying the disutility estimates obtained from the literature review, with $\mathrm{AE}$ incidence rates reported from the trial. ${ }^{7,8,26}$ Then, the weighted cumulative disutility was obtained by adding all the weighted disutility across AEs for each drug. Finally, a composite utility value was derived for RFS and PD states for both interventions by subtracting the weighted cumulative disutility from the base-case value (Table 2). Moreover, patients only received treatment while in the RFS state-once they transitioned to the PD state, treatment was discontinued. A 3\% discount rate per year was applied for both costs and utilities included in the model.

\section{MODEL ASSUMPTIONS}

For our model, based on clinical expert consultation, we assumed that the transition from the RFS state to PD state was $5 \%$ at all time points. A study by Liao et al estimated the transition probability for RFS to PD state to be $2 \%$ in the resected pancreatic cancer setting. ${ }^{25}$ Our assumption was more conservative, and to ensure the accuracy of the model, we ran it with $2 \%$ and $5 \%$ probability and found no change in the direction of the results.

We assumed that $\mathrm{AE}$ disutility and costs would be associated only for the 


\section{FIGURE 2 Tornado Diagram of 1-Way Sensitivity Analysis for FOLFIRINOX and GemNab}

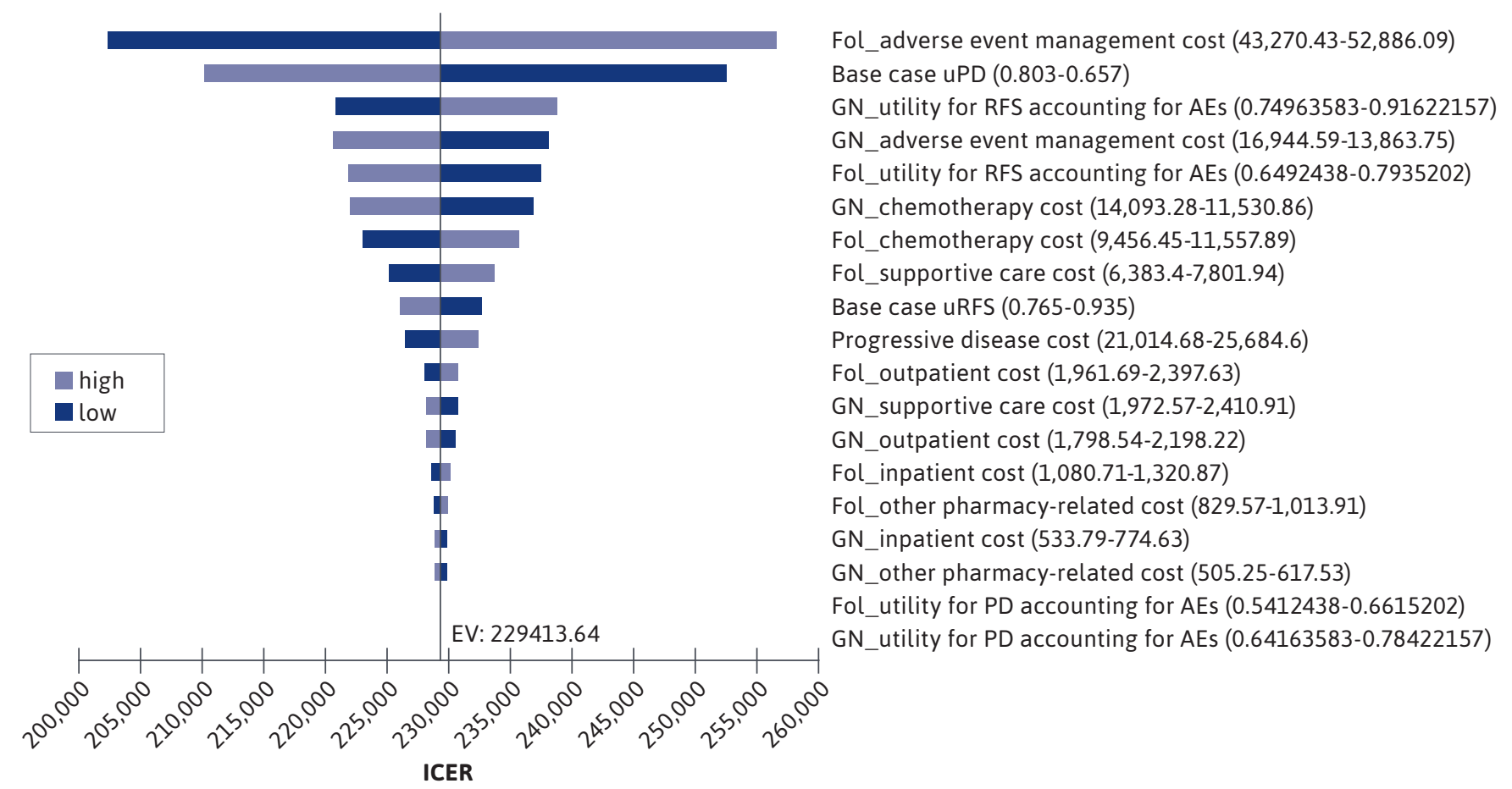

$A E s=$ adverse event; Fol =FOLFIRINOX; ICER =incremental cost-effectiveness ratio; $G N=$ gemcitabine nab-paclitaxel; $P D=$ progressive disease; $R F S=$ relapse-free survival; $u=$ utility.

first cycle, and then, base-case estimates would be used in the model. All patients entered the model in the RFS state, and AEs only occurred in the first cycle. This assumption is conservative, since significant AEs are likely to occur in early months. ${ }^{27}$ We consulted an oncology clinical pharmacist for an expert opinion and found that the majority of AEs occur early in or at the start of treatment. Assumption was therefore made to have AEs occur in the first month of treatment, since neither of the trials reported timing of the AEs.

\section{BASE-CASE AND SENSITIVITY ANALYSES}

CEA was performed to obtain total costs, quality-adjusted life-years (QALY), life-years (LY), and incremental costeffectiveness ratio (ICER). The primary outcome was the ICER, which is calculated as the ratio of difference in cost between each treatment strategy to the difference in effectiveness between each treatment strategy. All analyses were conducted using the TreeAge Pro 2020, HealthCare Version (TreeAge Software).
The effect of our assumptions on input parameters was explored with sensitivity analysis. One-way sensitivity analysis was conducted to determine the robustness of the model and to explore the influence of key input parameters. Ranges of parameter values were generated by using plus or minus $10 \%$ variation. Probabilistic sensitivity analysis (PSA) was also conducted to evaluate the simultaneous effect of uncertainty in model parameter values. In PSA, 1,000 iterations of the model were run. Each iteration used a separate value for input parameters that were drawn from prespecified distributions. A beta distribution was assumed for health utility values and probabilities, and a gamma distribution was assumed for costs. For health utilities where SDs were not available, a 10\% deviation of the mean values was assumed. The PSA generated a costeffectiveness acceptability curve (CEAC) that determined the cost-effectiveness of the 2 interventions at varying willingness-to-pay (WTP) thresholds. 


\begin{tabular}{|c|c|c|c|}
\hline Cost (per month) & FOLFIRINOX, $\$$ & GemNab, ${ }^{c} \$$ & Reference \\
\hline $\begin{array}{l}\text { Chemotherapy drug cost } \\
\text { (acquisition + administration) }\end{array}$ & 10,507 & 12,812 & 16 \\
\hline Supportive care cost & 7,092 & 2,191 & 16 \\
\hline Inpatient & 1,200 & 704 & 16 \\
\hline Outpatient & 2,179 & 1,998 & 16 \\
\hline Other pharmacy-related costs & 921 & 561 & 16 \\
\hline Adverse event management cost & 48,078 & 15,404 & 17,18 \\
\hline Cost for progressive disease & \multicolumn{2}{|c|}{23,349} & 20 \\
\hline Cost for death & \multicolumn{2}{|c|}{15,527} & 21 \\
\hline
\end{tabular}

\section{Results}

\section{BASE-CASE ANALYSIS}

In the base-case analysis, the estimated cost for FOLFIRINOX was $\$ 40,831$ higher than GemNab $(\$ 99,668$ vs $\$ 58,837)$. Despite the higher toxicity rate of FOLFIRINOX, the total effectiveness associated with FOLFIRINOX was higher (1.65 vs 1.47 QALYs). The RFS for both treatments was very similar (0.89 vs 0.88); however, FOLFIRINOX showed a slightly better gain in effectiveness in the PD state, with a utility value of 0.76 compared with 0.59 for GemNab. With respect to life expectancy, FOLFIRINOX was associated with an additional $0.25 \mathrm{LY}$ gained compared with GemNab (2.09 vs 1.84). The ICER for FOLFIRINOX compared with GemNab was $\$ 226,841$ per QALY and $\$ 163,325$ per LY (Table 3).

\section{SENSITIVITY ANALYSIS}

One-way sensitivity analysis results are displayed in the tornado diagrams using the ICER (Figure 2). The results were most sensitive to changes in $\mathrm{AE}$ management cost, base-case utility associated with PD, and utility of
RFS after accounting for AEs. The AE management cost for FOLFIRINOX and the utility value for PD played a vital role in model outcomes. When $\mathrm{AE}$ costs for FOLFIRINOX varied from $\$ 43,270$ to $\$ 52,886$, the ICER changed from $\$ 202,400$ to $\$ 256,426$, which was still higher than the WTP threshold of $\$ 200,000$ for this model. Base-case PD utility changes from 0.65 to 0.79 increased the ICER from $\$ 210,263$ per QALY to $\$ 253,000$ per QALY.

The remaining parameters in the model had minimal effect on the results. The CEAC obtained from PSA showed that FOLFIRINOX is not costeffective compared with GemNab at a WTP threshold of $\$ 200,000$ (Supplementary Figure 1, available in online article). The probability of FOLFIRINOX not being cost-effective at a WTP threshold of $\$ 200,000$ was $89.2 \%$. The PSA showed that mean (SD) costs, QALYs, and LYs gained for FOLFIRINOX were \$99,446 (\$2,779), 1.57 (0.17) QALYs, and 2.04 (0.12) LYs, and for GemNab, they were $\$ 58,768$ (\$1,423), 1.41 (0.15) QALYs, and 1.68 (0.09) LYs.

\section{Discussion}

To our knowledge, this is the first study to evaluate and compare the cost-effectiveness of FOLFIRINOX compared with GemNab as adjuvant treatment for resected pancreatic cancer in the United States. Our results show incremental costs of $\$ 40,831$ associated with FOLFIRINOX compared with GemNab, and total incremental effectiveness of 0.18 QALYS with the same QALY estimates for the RFS state (0.89 vs 0.88). From a clinical standpoint, such a slight increase in effectiveness for FOLFIRINOX may hold very little to no clinical value. We found that FOLFIRINOX was not cost-effective at a WTP threshold of $\$ 200,000$ per QALY. However, our results suggest that FOLFIRINOX would be cost-effective at a WTP threshold of $\$ 250,000$ per QALY or more. The most commonly used US WTP threshold value is $\$ 100,000$, but the WTP threshold for this study was $\$ 200,000$ per QALY, which was based on Ubel et al who stated that the US WTP threshold should be closer or equal to $\$ 200,000$ per QALY when inflation and WTP valuations are taken into consideration. ${ }^{28-30}$

The total monthly cost for FOLFIRINOX was approximately 1.7 times higher than the cost for GemNab. This higher cost could be attributed to significantly higher AE management costs of FOLFIRINOX because of its toxicity profile and slightly higher supportive care requirements, which include pharmacy, inpatient, and outpatient costs, compared with GemNab. ${ }^{16}$ Most of these costs were applicable to the RFS state, driving the RFS costs for FOLFIRINOX significantly higher than the PD state.

Although cost-effectiveness for adjuvant FOLFIRINOX compared with GemNab in resectable pancreatic cancer has not been previously reported, 
TABLE 2 Utility Input Parameter Values, Weighted Disutility for Adverse Events, and Weighted Composite Disutility Estimate for RFS and PD States

\begin{tabular}{|c|c|c|c|c|c|c|}
\hline A & \multicolumn{2}{|c|}{ B } & C & D & $\mathbf{E}$ & $\mathbf{F}$ \\
\hline Utility/disutility & $\begin{array}{c}\text { Base-case } \\
\text { estimate }\end{array}$ & $\begin{array}{l}\text { Base-case } \\
\text { reference }\end{array}$ & \multicolumn{2}{|c|}{ FOLFIRINOX } & \multicolumn{2}{|c|}{ GemNab } \\
\hline RFS state & 0.85 & 37 & \multirow[b]{3}{*}{$\begin{array}{l}\text { Study } \\
\text { estimate } \\
\text { for AE }\end{array}$} & \multirow[b]{3}{*}{$\begin{array}{c}\text { Disutility } \\
(B \times C)\end{array}$} & \\
\hline PD state & 0.73 & 37 & & & & \\
\hline Adverse event & $\begin{array}{l}\text { Base case } \\
\text { estimate }\end{array}$ & $\begin{array}{l}\text { Base-case } \\
\text { reference }\end{array}$ & & & $\begin{array}{l}\text { Study } \\
\text { estimate } \\
\text { for AE }\end{array}$ & $\begin{array}{c}\text { Disutility } \\
(B \times E)\end{array}$ \\
\hline Diarrhea & -0.288 & 26 & 0.186 & -0.054 & 0.028 & -0.008 \\
\hline Fatigue & -0.115 & 26 & 0.11 & -0.013 & 0.016 & -0.002 \\
\hline Neutropenia & -0.184 & 26 & 0.284 & -0.052 & 0.012 & -0.002 \\
\hline Thrombocytopenia & -0.184 & 26 & 0.013 & -0.002 & 0.012 & -0.002 \\
\hline Vomiting & -0.152 & 26 & 0.051 & -0.008 & 0.019 & -0.003 \\
\hline & & & Total & -0.129 & Total & -0.017 \\
\hline \multicolumn{3}{|c|}{ Weighted composite disutility } & \multicolumn{2}{|c|}{$\begin{array}{c}\text { FOLFIRINOX } \\
\text { (base-case } \\
\text { estimate-total) }\end{array}$} & \multicolumn{2}{|c|}{$\begin{array}{c}\text { GemNab } \\
\text { (base-case } \\
\text { estimate-total) }\end{array}$} \\
\hline \multicolumn{3}{|l|}{ RFS state } & \multicolumn{2}{|c|}{0.979} & \multicolumn{2}{|c|}{0.867} \\
\hline PD state & & & \multicolumn{2}{|c|}{0.859} & \multicolumn{2}{|c|}{0.747} \\
\hline
\end{tabular}

$A E=$ adverse event; $P D=$ progressive disease; $R F S=$ relapse-free survival.

our study findings are similar to previous studies by Gharaibeh et al, Zhoul et al, and Kurimoto et al, who found GemNab to be cost-effective compared with FOLFIRINOX in metastatic pancreatic cancer. ${ }^{17-19,31,32}$ The total cost in our study was $\$ 99,668$ and $\$ 58,837$ for FOLFIRINOX and GemNab, respectively. These estimates are slightly higher than the Gharaibeh et al study, where the estimated total costs of first-line FOLFIRINOX was $\$ 83,836$ and $\$ 54,843$ for GemNab. ${ }^{18}$ Compared with Gharaibeh's study, which reported costs in 2017 US dollars, the costs from our study are similar, and the slight increase could be a result of inflation to 2020 US dollars. Also, we included inpatient and outpatient costs associated with overall all-cause costs in the study, which was not accounted for in the Gharaibeh et al study.
The ICER for our study was $\$ 226,841$ per QALY and $\$ 163,325$ per LY gained. These estimates are lower than the 2 US studies conducted by Gharaibeh et al on first-line therapies for metastatic pancreatic cancer. ${ }^{17,18}$ The first study was an economic evaluation of GemNab and FOLFIRINOX, each compared with gemcitabine monotherapy (published in 2016). ${ }^{25}$ A second study evaluated the economics of several common systemic first-line treatments (published in 2018). ${ }^{18}$ We speculate on several reasons for the differences in ICER estimates between the Gharaibeh et al studies and our study.

First, differences in utility values between these studies could contribute to the lower ICER. The total effectiveness (ie, QALY and LY) for both Gharaibeh et al metastatic pancreatic cancer studies are lower than our estimates. ${ }^{17,18}$ The reason our effectiveness estimates are higher could be due to the study population. Our study focused on resectable pancreatic cancer, which is considered an early-stage cancer with first-line surgery therapy options. Surgical resection not only prolongs survival but also improves HRQoL. ${ }^{33}$

In contrast, metastatic pancreatic cancer is an advanced stage disease, and surgery is not a therapeutic option. Metastatic PC is associated with diminished QoL, ${ }^{34}$ which in turn reflects in the utility estimates. Also, our assumption of including $\mathrm{AE}$ data for costs and effectiveness only in the first cycle of the model could produce differences in estimates.

Finally, there could be methodological differences. Gharaibeh et al used HR estimates from indirect comparisons and included the proportional hazards assumption. ${ }^{24}$ We used the survival data from clinical trials, which could be a plausible contributor towards differences in the ICER.

It is important to note several important strengths of our study. In the absence of a direct head-to-head clinical trial, this is the only study that has performed an economic analysis of FOLFIRINOX and GemNab for resectable pancreatic cancer. The highest level of evidence was obtained directly from the PRODIGE-24 and APACT trials, where FOLFIRINOX and GemNab each showed survival benefits compared with gemcitabine monotherapy. There were no significant differences in baseline characteristics for both trials. HR estimates for survival outcomes were not used. Instead, survival curves from the trials were used and parametric survival fitting was done. This helped us to generalize the findings and extrapolate survival results beyond the data horizon. Also, this study accounted for important 


\begin{tabular}{|c|c|c|}
\hline Parameter & FOLFIRINOX & GemNab \\
\hline \multicolumn{3}{|l|}{ Cost, \$ } \\
\hline RFS & 71,522 & 34,904 \\
\hline PD & 28,146 & 23,932 \\
\hline Total & 99,668 & 58,837 \\
\hline Incremental costs, \$ & & 40,831 \\
\hline \multicolumn{3}{|l|}{ Effectiveness, QALY } \\
\hline RFS & 0.89 & 0.88 \\
\hline PD & 0.76 & 0.59 \\
\hline Total & 1.65 & 1.47 \\
\hline Incremental QALY & 0.18 & \\
\hline ICER, \$ per QALY & \multicolumn{2}{|c|}{226,841} \\
\hline \multicolumn{3}{|l|}{ LYs } \\
\hline RFS & 1.06 & 1.03 \\
\hline PD & 1.03 & 0.81 \\
\hline Total & 2.09 & 1.84 \\
\hline Incremental LYs & 0.25 & \\
\hline ICER, \$ per LY & \multicolumn{2}{|c|}{163,325} \\
\hline
\end{tabular}

grade $3 / 4$ AEs, while incorporating costs and effectiveness estimates in the model.

\section{LIMITATIONS}

There are several limitations that need to be considered for our study. The PRODIGE-24 and APACT trials did not measure QoL utility data. First, we obtained costs and utility values from published literature ${ }^{24,25}$ and inflated the costs to 2021 US dollar values using the Consumer Price Index (CPI). However, CPI adjustments do not account for various disease parameters (eg, different location, site complexity, disease complexity, patient access, and treatment completion) that influence costs; it only accounts for inflation. Using directly published cost and utility estimates is always associated with some level of uncertainty about study design and bias, and we recognize that using national data would have surely added more value to the study.

Second, because of lack of direct head-to-head trials comparing FOLFIRINOX and GemNab, this CEA analysis used effectiveness estimates directly from the trials. Realworld comparative evidence for resected pancreatic cancer is sparse; therefore, we were limited to using trial data.
Third, the rate of natural progression was conservatively assumed to be $5 \%$. However, we explored the sensitivity of our model to this assumption by also running the model using a value of $2 \%$ for this input and found no change in the direction of the results.

Fourth, we assumed health care costs in the metastatic population to be similar to resectable pancreatic patients because limited evidence exists for costs associated with resected pancreatic cancer. A study by O'Neil et al calculated the direct medical cost of care among Medicare enrollees for resected pancreatic cancer but provided no information on costs associated with adjuvant treatment. ${ }^{35}$ Another study by Cerullo et al estimated the median total chemotherapy payment associated with adjuvant treatment using the IBM MarketScan database between 2010 and $2014 .{ }^{36}$ The breakdown for other costs such as inpatient, outpatient, and supportive care was not provided, and there is no literature to support these costs in the resectable setting. Therefore, we used metastatic estimates for this study.

Fifth, nonserious grade 1/2 AEs were excluded from the analysis due to low prevalence in the trials. However, serious grade $3 / 4 \mathrm{AEs}$ are considered to significantly affect survival and QoL. Therefore, we expect that exclusion of $1 / 2$ AEs minimally affected the economic evaluation.

Sixth, PRODIGE-24 trial was conducted in the United States, France, and Canada, whereas APACT was conducted exclusively outside the United States. These geographical differences could have potentially influenced HR estimates in the trial; also, the approach to treat AEs within each trial could be different, which in turn could affect the clinical outcomes and our CEA estimates.

Finally, we analyzed our model only from the US health care perspective. The societal perspective was not assessed and could be an important focus for future research.

\section{Conclusions}

To our knowledge, this is the first study to demonstrate the cost-effectiveness of FOLFIRINOX compared with GemNab as postsurgery adjuvant treatment for resected pancreatic cancer. The results demonstrated that FOLFIRINOX is not cost-effective compared with GemNab at a WTP threshold of $\$ 200,000$ per QALY from a US payer perspective. AE-related costs and disutility values might be an important factor to consider in this CEA of FOLFIRINOX vs GemNab.

The findings of this study will help health care providers and policymakers in guiding decision making and guideline development. The decision of which treatment to use should be left to the patients and their providers. Patient characteristics such as overall health and functional status 
and ability to tolerate AEs may affect their decisions. PRODIGE and APACT showed some variation in ECOG performance status (Supplementary Table 3 , available in online article), which suggests that slightly healthier patients received GemNab as opposed to FOLFIRINOX. Also, it is important for policymakers to consider WTP thresholds; if the threshold is not increased, FOLFIRINOX will not be considered cost-effective. From a clinical standpoint, the incremental effectiveness associated with FOLFIRINOX was very modest and holds little clinical significance; therefore, decisions for selecting optimal treatment option should assess the tradeoff between patient characteristics, payments, and survival.

Future research should focus on collecting and analyzing real-world data for cost and QoL measures. Because of the increasing demand for CEAs and the unrestricted methods used to conduct such studies, emphasis on including real-world data elements, such as data on rebates and copays, can help improve generalizability and precision of costeffectiveness estimates.

\section{DISCLOSURES}

This research received no specific grant from any funding agency in the public, commercial, or not-for-profit sectors. The authors have no conflicts of interest to declare.

\section{REFERENCES}

1. Rawla P, Sunkara T, Gaduputi V. Epidemiology of pancreatic cancer: global trends, etiology and risk factors. World J Oncol. 2019;10(1):10-27. doi:10.14740/ wjon1166
2. Reynolds K. Pancreatic cancer still on path to become second leading cause of cancer-related death in US by 2020. Pancreatic Cancer Action Network. January 8, 2019. Accessed September 9, 2020. https:// www.pancan.org/press-releases/ pancreatic-cancer-still-on-path-tobecome-second-leading-cause-of-cancerrelated-death-in-u-s-by-2020/

3. American Cancer Society. Key statistics for pancreatic cancer. Accessed September 9, 2020. https://www.cancer. org/cancer/pancreatic-cancer/about/ key-statistics.html

4. Pancreatic Cancer Action Network. What is pancreatic cancer? Accessed September 9, 2020. https://www. pancan.org/facing-pancreaticcancer/about-pancreatic-cancer/ what-is-pancreatic-cancer/

5. Chakraborty S, Singh S. Surgical resection improves survival in pancreatic cancer patients without vascular invasion-a population based study. Ann Gastroenterol Q Publ Hell Soc Gastroenterol. 2013;26(4):346-52.

6. Neoptolemos JP, Palmer DH, Ghaneh P, et al. Comparison of adjuvant gemcitabine and capecitabine with gemcitabine monotherapy in patients with resected pancreatic cancer (ESPAC-4): a multicentre, open-label, randomised, phase 3 trial. Lancet. 2017;389(10073):1011-24. doi: 10.1016/S0140-6736(16)32409-6

7. Conroy T, Hammel P, Hebbar M, et al. FOLFIRINOX or gemcitabine as adjuvant therapy for pancreatic cancer. N Engl J Med. 2018;379(25):2395-406. doi: 10.1056/ NEJMoa1809775.

8. Tempero A, Reni M, Hanno R, et al. APACT: phase III, multicenter, international, open-label, randomized trial of adjuvant nab-paclitaxel plus gemcitabine (nab-P/G) vs gemcitabine (G) for surgically resected pancreatic adenocarcinoma. J Clin Oncol. 2019;37(15 Suppl):4000. Accessed September 9, 2020. https://ascopubs.org/doi/abs/10.1200/ JCO.2019.37.15 suppl.4000
9. Galvano A, Castiglia M, Rizzo S, et al. Moving the target on the optimal adjuvant strategy for resected pancreatic cancers: a systematic review with metaanalysis. Cancers (Basel). 2020;12(3):534. doi:10.3390/cancers12030534

10. National Comprehensive Cancer Network. NCCN Evidence Blocks. Pancreatic adenocarcinoma, version 2.2021. Accessed August 29, 2021. https://www.nccn.org/guidelines/ guidelines-with-evidence-blocks

11. Mariotto AB, Enewold L, Zhao J, Zeruto CA, Yabroff KR. Medical care costs associated with cancer survivorship in the United States. Cancer Epidemiol Biomarkers Prev. 2020;29(7):1304-12. doi:10.1158/1055-9965.EPI-19-1534

12. Soefje SA. Managing the economic impact of advanced pancreatic cancer. Am J Manag Care. 2019;25(1 Suppl):S11-S16. Accessed February 11, 2021. https://www. ajmc.com/view/managing-the-economicimpact--of-advanced-pancreatic-cancer

13. Fountzilas C. Updates in the management of GI malignancies. Video. Clinical oncology: year 2019 in review. Roswell Park Comprehensive Cancer Center. 2019. Accessed August 29, 2021. https://physicianresources. roswellpark.org/symposium/clinical-oncology-year-2019-in-review/ flatDisplayPageSymposium\#BA1 4CB20-F0E6-11E9-8FDAFAE29D4FB66B

14. Hoyle MW, Henley W. Improved curve fits to summary survival data: application to economic evaluation of health technologies. BMC Med Res Methodol. 2011;11(1):139. doi:10.1186/1471-2288-11-139

15. Bounthavong M. Generating survival curves from study data: an application for Markov models (Part 2 of 2). March 15, 2018. Accessed October 12, 2020. https:// mbounthavong.com/blog/category/ Cost-effectiveness+models 
16. Kim GP, Parisi MF, Patel MB, Pelletier CL, Belk KW. Comparison of treatment patterns, resource utilization, and cost of care in patients with metastatic pancreatic cancer treated with first-line nab-paclitaxel plus gemcitabine or FOLFIRINOX. Expert Rev Clin Pharmacol. 2017;10(5):559-65. doi:10.1080/ 17512433.2017.1302330

17. Gharaibeh M, McBride A, Bootman JL, Patel H, Abraham I. Economic evaluation for the US of nab-paclitaxel plus gemcitabine versus FOLFIRINOX versus gemcitabine in the treatment of metastatic pancreas cancer. J Med Econ. 2017;20(4):345-52. doi:10.1080/13696998.2 016.1269015

18. Gharaibeh M, McBride A, Alberts DS, et al. Economic evaluation for USA of systemic chemotherapies as first-line treatment of metastatic pancreatic cancer. Pharmacoeconomics. 2018;36(10):1273-84. doi:10.1007/ s40273-018-0678-6

19. Gharaibeh M, McBride A, Bootman JL, Abraham I. Economic evaluation for the UK of nab-paclitaxel plus gemcitabine in the treatment of metastatic pancreas cancer. Br J Cancer. 2015;112(8):1301-05. doi: 10.1038/bjc.2015.65

20. Chang S, Long SR, Kutikova L, Bowman L, Crown WH, Lyman GH. Burden of pancreatic cancer and disease progression: economic analysis in the US. Oncology. 2006;70(1):71-80. doi:10.1159/000091312

21. Scitovsky AA. "The high cost of dying": what do the data show? Milbank Q. 2005;83(4):825-41. doi:10.1111/j.1468-0009.2005.00402.x

22. US Bureau of Labor Statistics. Consumer Price Index. Accessed October 12, 2020. https://www.bls.gov/cpi/

23. US Bureau of Labor Statistics. CPI inflation calculator. Accessed October 12, 2020. https://www.bls.gov/data/inflation calculator.htm
24. Huang J, Liao W, Zhou J, et al. Cost-effectiveness analysis of adjuvant treatment for resected pancreatic cancer in China based on the ESPAC-4 trial. Cancer Manag Res. 2018;10:4065-72. doi:10.2147/CMAR.S172704

25. Liao W, Huang J, Zhu G, et al. S-1 or gemcitabine adjuvant therapy in resected pancreatic cancer: a cost-effectiveness analysis based on the JASPAC-01 trial. Expert Rev Pharmacoecon Outcomes Res. 2020;20(1):133-38. doi:10.1080/14737167.20 20.1677155

26. Attard CL, Brown S, Alloul K, Moore MJ. Cost-effectiveness of folfirinox for first-line treatment of metastatic pancreatic cancer. Curr Oncol. 2014;21(1):e41-e51. doi:10.3747/co.21.1327

27. van Holstein Y, Kapiteijn E, Bastiaannet E, van den Bos F, Portielje J, de Glas NA. Efficacy and adverse events of immunotherapy with checkpoint inhibitors in older patients with cancer. Drugs Aging. 2019;36(10):927-38. doi:10.1007/ s40266-019-00697-2

28. Claxton K, Martin S, Soares M, et al. Appendix 1: Systematic review of the literature on the cost-effectiveness threshold. In Claxton K, Martin S, Soares M, et al. Methods for the Estimation of the National Institute for Health and Care Excellence CostEffectiveness Threshold. Health Technology Assessment No. 19. NIHR Journals Library; 2015. Accessed February 11, 2021. https://www.ncbi.nlm.nih.gov/ books/NBK274312/

29. Ubel PA, Hirth RA, Chernew ME, Fendrick AM. What is the price of life and why doesn't it increase at the rate of inflation? Arch Intern Med. 2003;163(14):1637-41. doi:10.1001/ archinte.163.14.1637

30. Cameron D, Ubels J, Norström F. On what basis are medical costeffectiveness thresholds set? Clashing opinions and an absence of data: a systematic review. Glob Health Action. 2018;11(1):1447828. doi:10.1080/16549716.20 18.1447828
31. Zhou J, Zhao R, Wen F, et al. Cost-effectiveness analysis of treatments for metastatic pancreatic cancer based on Prodige and MPACT trials. Tumori. 2016;2016(3):294-300. doi: 10.5301/ tj.5000499

32. Kurimoto M, Kimura M, Usami E, et al. Comparing the cost-effectiveness of FOLFIRINOX, nab-paclitaxel plus gemcitabine, gemcitabine and S-1 for the treatment of metastatic pancreatic cancer. Mol Clin Oncol. 2017;7(1):125-30. doi:10.3892/mco.2017.1278

33. Crippa S, Domínguez I, Rodríguez JR, et al. Quality of life in pancreatic cancer: analysis by stage and treatment. J Gastrointest Surg Off J Soc Surg Aliment Tract. 2008;12(5):783. doi:10.1007/ s11605-007-0391-9

34. Laquente B, Macarulla T, Bugés C, et al. Quality of life of patients with metastatic pancreatic adenocarcinoma initiating first-line chemotherapy in routine practice. BMC Palliat Care. 2020;19(1):103. doi: 10.1186/ s12904-020-00610-4.

35. O'Neill CB, Atoria CL, O'Reilly EM, LaFemina J, Henman MC, Elkin EB. Costs and trends in pancreatic cancer treatment. Cancer. 2012;118(20):5132-39. doi:10.1002/cncr.27490

36. Cerullo M, Gani F, Chen SY, et al. Assessing the financial burden associated with treatment options for resectable pancreatic cancer. Ann Surg. 2018;267(3):544-51. doi:10.1097/ sla.0000000000002069

37. Huang J, Liao W, Zhou J, et al. Cost-effectiveness analysis of adjuvant treatment for resected pancreatic cancer in China based on the ESPAC-4 trial. Cancer Manag Res. 2018;10:4065-72. doi:10.2147/CMAR.S172704 